\title{
Evaluation of the stiffness characteristics of rapid palatal expander screws
}

\author{
Luca Lombardo ${ }^{1}$, Enrico Sacchi², Maria Larosa ${ }^{1 *}$, Francesco Mollica ${ }^{3}$, Valentina Mazzanti $^{3}$, \\ Giorgio Alfredo Spedicato ${ }^{4}$ and Giuseppe Siciliani ${ }^{1}$
}

\begin{abstract}
Background: The aim of this study is to evaluate the mechanical properties of the screws used for rapid expansion of the upper jaw.

Methods: Ten types of expansion screw were assessed, seven with four arms: Lancer Philosophy 1, Dentaurum Hyrax Click Medium, Forestadent Anatomic Expander type "S", Forestadent Anatomic Expander type "S" for narrow palates, Forestadent Memory, Leone A 2620-10 with telescopic guide, and Leone A 0630-10 with orthogonal arms; and three with two arms: Dentaurum Variety S.P., Target Baby REP Veltri, and Leone A 362113. A test expander with the mean dimensions taken from measurements on a sample of 100 expanders was constructed for each screw. The test expanders were connected to the supports of an Instron 4467 (Instron Corp., USA) mechanical testing machine equipped with a $500 \mathrm{~N}$ load cell, and the compression force exerted after each activation was measured. The mean forces expressed by the two- and four-arm expanders were then compared.
\end{abstract}

Results: After five activations, the forces expressed by the two-arm devices were double than those expressed by the four-arm devices on average ( $224 \pm 59.9 \mathrm{~N}$ vs. $103 \pm 32.9 \mathrm{~N})$, and such values remained high after subsequent activations.

Conclusions: The expanders tested demonstrated stiffness characteristics compatible with opening of the palatine sutures in pre-adolescent patients. The stiffness of such devices can be further increased during the construction phase.

\section{Background}

Normalizing the dimensions of the upper jaw is of primary importance in orthodontics. In fact, an upper jaw of incorrect dimensions may affect both the transversal and sagittal planes [1]. A rapid palatal expander (RPE) is the most popular device of choice in this regard, characterized by safety, predictability, and efficiency [2-8].

RPEs have a predominantly orthopedic action, although they do bring about a certain degree of dental expansion, in turn provoking labial inclination of the teeth. This effect becomes more pronounced as age advances, since an increase in the interdigitation at the palatine suture increases its resistance to opening and reduces the orthopedic effects in favor of dental effects [9-12].

Indeed, the change in skeletal transversal dimensions decreases from $50 \%$ to roughly a third of the quantity of

\footnotetext{
* Correspondence: dott.marialarosa@gmail.com

${ }^{1}$ Postgraduate School of Orthodontics, University of Ferrara, Via Montebello

31, 44121 Ferrara, Italy

Full list of author information is available at the end of the article
}

RPE screw activation after the pubertal growth peak in initial permanent dentition [13].

Rapid maxillary expansion treatment is able to induce more pronounced transverse craniofacial changes at the skeletal level before the peak in skeletal growth, and skeletal outcomes of greater magnitude and stability can be obtained when the expander is used before the pubertal growth spurt. When RME therapy is performed after the pubertal peak, on the other hand, transverse changes shift to the dentoalveolar level $[14,15]$.

Various models of screws and operating protocols have been suggested, both for achieving standard expansion and for activating the premaxillary sutures via alternate phases of expansion and contraction [16-19]. In order to open the median palatine suture to a sufficient degree and contemporaneously avoid a significant dentoalveolar response, RPEs must exert intense levels of force within a short time-frame. Hence, they must possess sufficient stiffness characteristics to enable them to exert such forces without deformation, so as to minimize the inclination of 
the teeth $[20,21]$. Furthermore, the stiffness characteristics of an expander must be increased when a patient presents with a particularly deep palate [22].

In this regard, the use of miniscrews to stabilize RPEs seems to be helpful, especially in late adolescence, and is currently the focus of ongoing research [23, 24]. However, to date, available data is scarce. For example, Muchitsch et al. [25] analyzed only the mechanical characteristics of the arms of RPEs, while Camporesi et al. [26] analyzed the compressive forces developed at each activation of three types of expander screw.

With a view to reducing patient discomfort and facilitating oral hygiene procedures, manufacturers are developing and marketing increasingly less bulky, more streamlined RPEs [27, 28], and we set out to evaluate the stiffness characteristics of several such devices.

\section{Methods}

The experiment evaluated 10 of the rapid expansion screws found on the market, all in medical-grade stainless steel (see Table 1 for details). Seven of the expansion screws had four retention arms and three had two retention arms. Each was welded to orthodontic bands and evaluated for overall stiffness, which comprised not only the stiffness of the screws themselves but also the resistance of the entire structure, including the welded joints. The deformation of the screws alone was not tested, as enormous forces would need to be measured.

\section{RPE construction}

Each screw was used in the construction of an RPE modeled on average values derived from measurements made on 100 expanders constructed to fit 100 Caucasian patients ( 54 females and 46 males) aged between 8 and 13. All patients of the sample needed a RPE treatment. The patients already treated by orthodontics were excluded. This age range was chosen because the RPE allows favorable orthopedic changes and it is widely utilized by patients of this age.

The measurements made on these 100 RPEs were as follows: (1) length of the anterior arms comprising the screw body; (2) length of the posterior arms comprising the screw body; (3) bend angle of the anterior arms; and (4) bend angle of the posterior arms. A copper wire shaped to fit the morphology of the retention arms was used to perform these measurements, which were made using a goniometer (angular measurements) and gauge (linear measurements). All measurements were performed by the same operator, and the sample means calculated are reported in Table 2.

Two metal wires (one for the anterior arms and one for the posterior arms) of $0.8-\mathrm{mm}$ diameter were used to transfer these values to a plaster model of standard upper arch (Fig. 1). A set-up was performed to adapt the standard arch form to the means obtained from our measurements.

The first molars were removed from these models and replaced with analogous metal teeth, joined together by means of a threaded pin to ensure that they remained parallel and that the RPEs constructed around them would be correctly aligned with the mechanical testing machine; an Instron 4467 (Instron Corp., USA) with $500-\mathrm{N}$ load cell was to be used for the stiffness testing.

Before testing, the metal teeth were fixed to the plaster model using wax to create a master model, duplicated

Table 1 Screw characteristics

\begin{tabular}{|c|c|c|c|c|c|}
\hline Two-arm screws & $\begin{array}{l}\text { Max. } \\
\text { expansion }\end{array}$ & $\begin{array}{l}\text { Arm } \varnothing \\
(\mathrm{mm})\end{array}$ & $\begin{array}{l}\text { Screw body size } \\
(\mathrm{mm})\end{array}$ & $\begin{array}{l}\text { Amount of expansion per activation } \\
(\mathrm{mm})\end{array}$ & Lot no. \\
\hline Dentaurum Variety S.P. two-arms & 12 & 1.48 & $9.6 \times 5 \times 3$ & 0.8 & 435299 \\
\hline Veltri Target baby REP & 13 & 1.45 & $11 \times 6 \times 4.5$ & 0.8 & 700032 \\
\hline Leone A 362113 two-arms & 13 & 1.48 & $10 \times 6 \times 4.5$ & 0.8 & 12032901 \\
\hline Four-arm screws & $\begin{array}{l}\text { Max. } \\
\text { expansion }\end{array}$ & $\begin{array}{l}\operatorname{Arm} \varnothing \\
(\mathrm{mm})\end{array}$ & $\begin{array}{l}\text { Screw body size } \\
(\mathrm{mm})\end{array}$ & $\begin{array}{l}\text { Amount of expansion per activation } \\
(\mathrm{mm})\end{array}$ & Lot $n^{\circ}$ \\
\hline Lancer Philosophy 1 & 10 & 1.55 & $8 \times 8 \times 3.5$ & 0.8 & $\begin{array}{l}\text { RPE } \\
\text { OOO440 }\end{array}$ \\
\hline Dentaurum Hyrax Click Medium & 10 & 1.48 & $10 \times 11 \times 4$ & 0.8 & 435361 \\
\hline Forestadent Anatomic Exp. Type "S" & 10 & 1.48 & $12 \times 7 \times 3.5$ & 0.8 & 48297815 \\
\hline $\begin{array}{l}\text { Forestadent Type "S" for narrow } \\
\text { palates }\end{array}$ & 10 & 1.48 & $12 \times 7 \times 3.5$ & 0.8 & 7399006 \\
\hline Forestadent Memory & 10 & 1.48 & $15 \times 10 \times 4$ & 0.8 & 14957593 \\
\hline $\begin{array}{l}\text { Leone A 2620-10 with telescopic } \\
\text { guides }\end{array}$ & 10 & 1.48 & $14 \times 11 \times 4$ & 0.8 & 12122001 \\
\hline $\begin{array}{l}\text { Leone A 0630-10 with orthogonal } \\
\text { arms }\end{array}$ & 10 & 1.48 & $10 \times 6 \times 4.5$ & 0.8 & 13011601 \\
\hline
\end{tabular}


Table 2 Means of measures

\begin{tabular}{lll}
\hline 1. Measurement & Kind of measure & Mean value \\
\hline & Length of posterior arms (including screw body) & $41.3 \mathrm{~mm}$
\end{tabular}
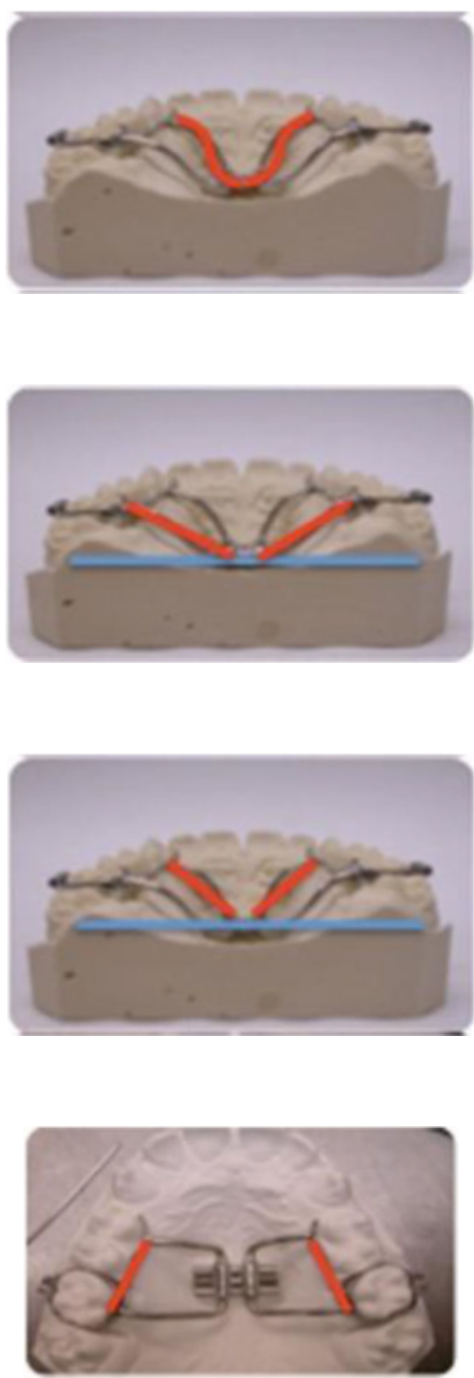

for the construction of each test RPE to ensure that they all had identical form (Figs. 2 and 3). The parameters used in the construction of the test devices are also reported in Table 2.

Orthodontic bands (LEONE MOD. E8305 no. 14) were then fitted to the first molars of each model, and the test screw brazewelded on the bands. The RPE constructed for the four-arm screw featured palatal supports, and no bands on the premolars. The two-arm RPEs were welded to the first molars and featured no palatal supports. The constructed RPEs were bonded to the metal teeth using composite cement (ULTRA BAND-LOCK, Reliance, USA) and light-cured using an LED curing light (Elipar Freelight 2, 3M Espe, 


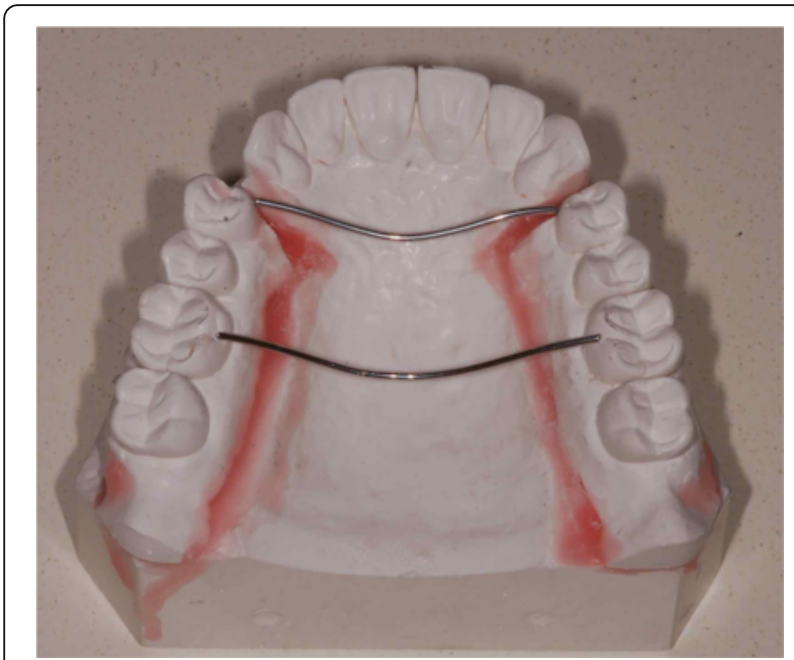

Fig. 1 Transferring the mean values measured to the plaster models

wavelength $430 \div 480 \mathrm{~nm}$, intensity $1200 \mathrm{~mW} / \mathrm{mm}^{2}$ ) for $30 \mathrm{~s}$ from the occlusal surface, the most effective method of bonding bands with this type of bonding agent [29].

\section{RPE activation procedure}

The RPEs were fixed to the Instron machine via rigid supports connected to the metal teeth (Fig. 4). Each RPE was then activated by the key provided so that the point of application of the force on the teeth lay on the long axis of the support. The compression force expressed after each activation was measured until either the maximum separation capacity of the screw had been reached or the activation key had deformed. The means and standard deviations of the forces expressed by the twoand four-arm RPEs after the activations were calculated and compared.

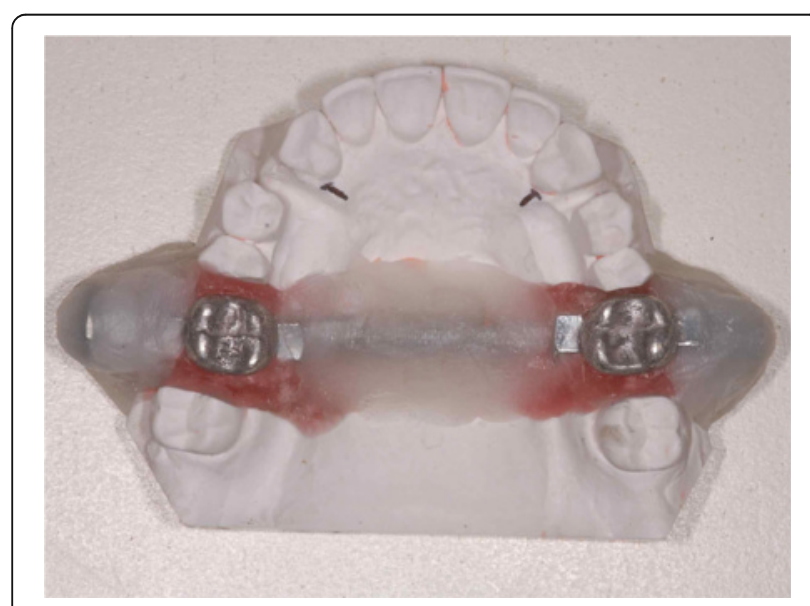

Fig. 2 Master model featuring metal teeth

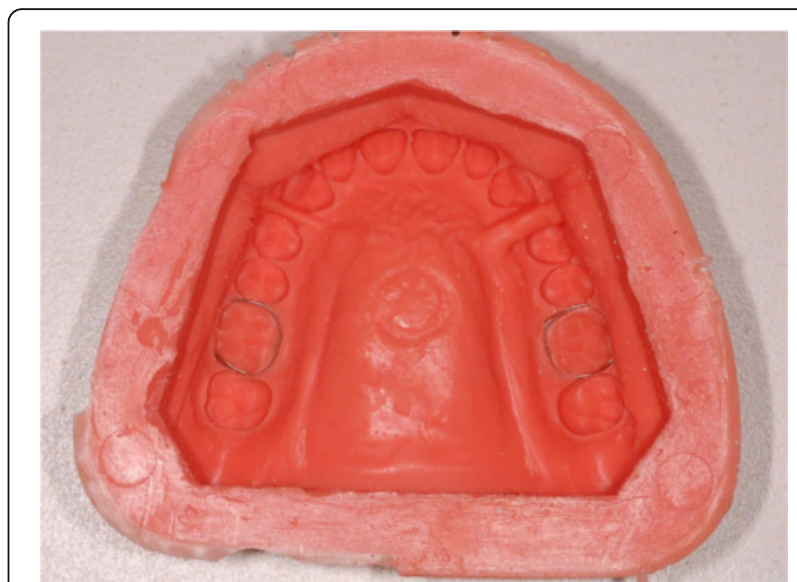

Fig. 3 Duplication of the master model in silicone

\section{Statistical analysis}

The statistical analysis aimed to assess how type (two arms, $2 \mathrm{~b}$, or four arms, 4b) and activation $(1,2, \ldots, 26)$ influence the measured strength. It was performed on 10 unique models that were measured for up to 26 activations (the effective length of measurement varied by model) and the analysis was performed using the growth curve analysis [30] approach. In particular, the strength behavior upon activation was approximated using a three-degree polynomial as a function of activation (baseline model). Polynomial's coefficients vary by type. To confirm the main conclusions $t$, a repeated measures ANOVA on the activation range where both $2 \mathrm{~A}$ and $2 \mathrm{~B}$ models were measured $(1,2, \ldots, 11)$ consistently.

The polynomial functional expression of the baseline model is:

$$
\begin{aligned}
\text { strength }_{i t}= & \alpha_{i}+4 b_{i}+\beta \times t+\beta_{4 b} \times \mathrm{t}+\gamma \times \mathrm{t}^{2}+\gamma_{4 b} \\
& \times t^{2}+\delta \times t^{3}+\delta_{4 \mathrm{~b}} \times t^{3}+\varepsilon_{\mathrm{it}}
\end{aligned}
$$

Specifically, the strength values observed at time $t$ on the $i$ th model are as follows:

- A model-specific intercept (treated as random effect), indicating material specific strength response tendency

- A constant intercept that applies when the model is $4 \mathrm{~b}$ type, indicating the baseline variation of strength due for the sample being of $4 \mathrm{~b}$ type

- The terms $\beta, \gamma$, and $\delta$, respectively, represent the first-, second-, and third-degree polynomial coefficients and are supplementary polynomial terms that applies when the model is $4 \mathrm{~b}$. The cubic curve tries to approximate the non-linear behavior of strength against activation, while the $4 \mathrm{~b}$ terms represent interaction terms that allow the polynomial shape to change 


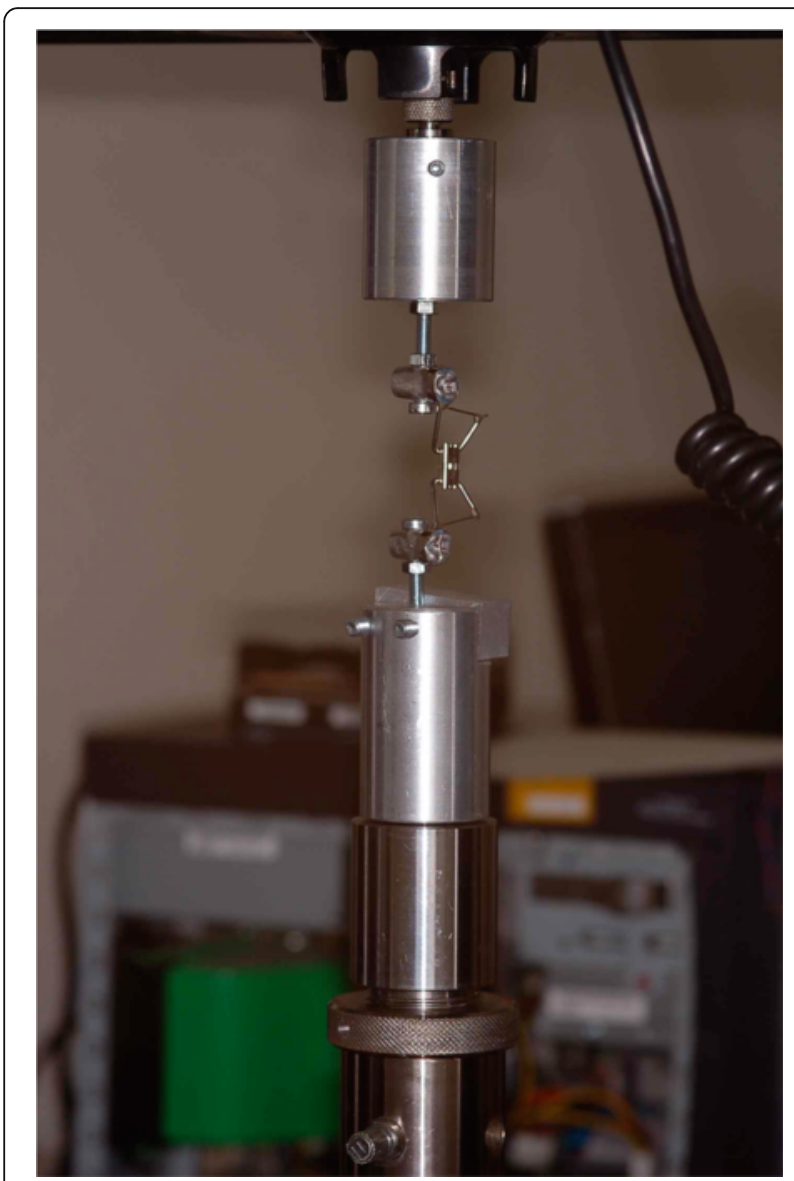

Fig. 4 Test RPE attached to the Instron machine aligned with the major axis of the supports between $4 \mathrm{a}$ and $4 \mathrm{~b}$ models which represent an additive error term. The repeated measures ANOVA assumed the type variable as the "between" factor and the activation (levels ranging from 1 to 11 ) as the "within" factor. A post hoc analysis has been therefore performed to compare difference in strength means between $4 \mathrm{~b}$ and $2 \mathrm{~b}$ types by activation level.

The statistical significance was assessed using a $5 \%$ threshold. R software [31] was used throughout the data processing, and the lme4 $\mathrm{R}$ software package [32] was used to estimate both the growth curve and the repeated measures ANOVA. Post hoc analysis was performed using lsmeans $\mathrm{R}$ package [33].

\section{Results}

Figure 5 shows the results of the stiffness of the complete RPEs welded to the bands; the $x$ axis shows the number of activations, and the $y$ axis the force expressed in Newtons. The curves terminate at the point at which further activation was not possible due to screw block. The activation keys started to show signs of deformation around the 150-N mark. The greater the stiffness of the RPE (the slope of the activation/force curve), the greater the force at a particular activation, and as the graph shows, the two-arm RPEs present a far steeper curve than the four-arm devices and express considerable forces even from the initial activations, reaching maximum values of $288 \mathrm{~N}$ (Leone A3621-13), $302 \mathrm{~N}$ (Dentaurum Variety SP), and $303 \mathrm{~N}$ (Target Baby REP), respectively. In each case, after the peak, the deformation of the retention arms and screw body prevented further increases in force. In contrast, the force per activation of the four-arm screws was smaller, as seen by the flatter, more regular curves on the graph. In particular,

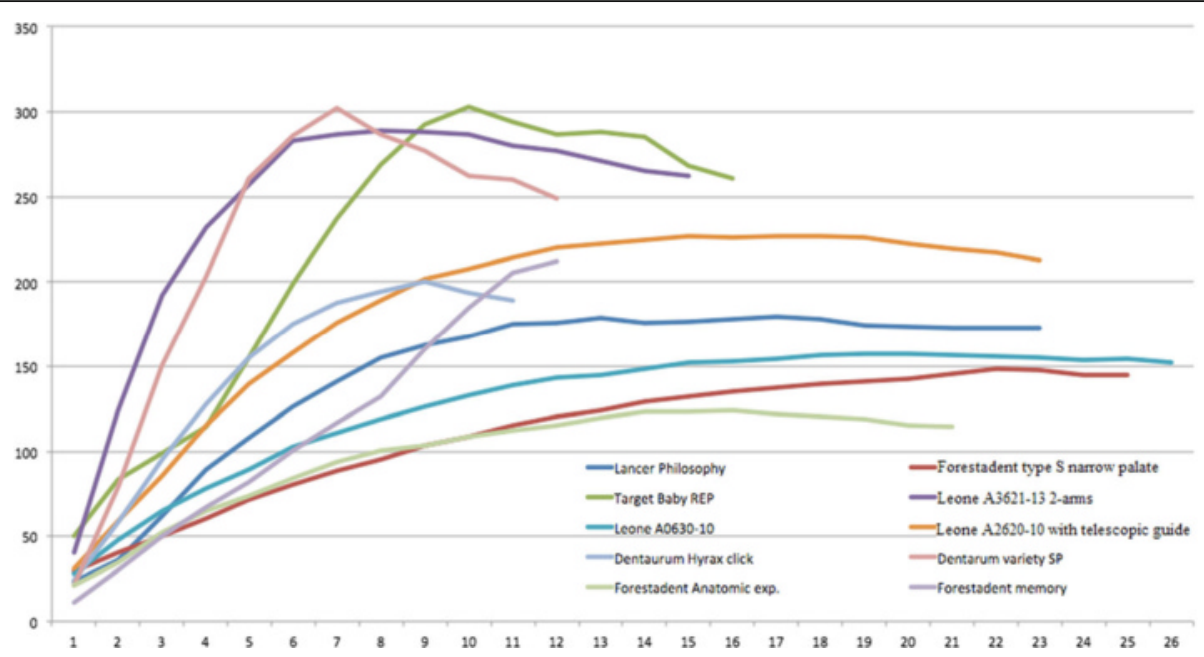

Fig. 5 Results of the RPE stiffness tests 
Table 3 Results of stiffness tests

\begin{tabular}{|c|c|c|c|c|}
\hline Type & Activations & Number & $\mathrm{Mu}$ & SD \\
\hline $2 B$ & 1 & 3 & 37 & 14.6 \\
\hline $2 B$ & 2 & 3 & 96 & 24.7 \\
\hline $2 B$ & 3 & 3 & 147 & 46.6 \\
\hline $2 B$ & 4 & 3 & 183 & 61.3 \\
\hline $2 B$ & 5 & 3 & 224 & 59.9 \\
\hline $2 \mathrm{~B}$ & 6 & 3 & 256 & 49.7 \\
\hline $2 \mathrm{~B}$ & 7 & 3 & 275 & 34.0 \\
\hline $2 B$ & 8 & 3 & 282 & 11.0 \\
\hline $2 B$ & 9 & 3 & 286 & 8.2 \\
\hline $2 B$ & 10 & 3 & 284 & 20.7 \\
\hline $2 B$ & 11 & 3 & 278 & 17.1 \\
\hline $2 B$ & 12 & 3 & 271 & 19.7 \\
\hline $2 B$ & 13 & 3 & 280 & 12.0 \\
\hline $2 B$ & 14 & 3 & 275 & 14.1 \\
\hline $2 B$ & 15 & 3 & 265 & 4.2 \\
\hline $4 \mathrm{~B}$ & 1 & 7 & 24 & 6.6 \\
\hline $4 \mathrm{~B}$ & 2 & 7 & 44 & 11.6 \\
\hline $4 B$ & 3 & 7 & 66 & 18.0 \\
\hline $4 \mathrm{~B}$ & 4 & 7 & 86 & 26.2 \\
\hline $4 \mathrm{~B}$ & 5 & 7 & 103 & 32.9 \\
\hline $4 \mathrm{~B}$ & 6 & 7 & 118 & 36.8 \\
\hline $4 B$ & 7 & 7 & 131 & 39.2 \\
\hline $4 \mathrm{~B}$ & 8 & 7 & 141 & 40.1 \\
\hline $4 \mathrm{~B}$ & 9 & 7 & 151 & 41.6 \\
\hline $4 \mathrm{~B}$ & 10 & 7 & 158 & 40.9 \\
\hline $4 \mathrm{~B}$ & 11 & 7 & 164 & 42.2 \\
\hline $4 B$ & 12 & 7 & 165 & 45.4 \\
\hline $4 \mathrm{~B}$ & 13 & 7 & 158 & 42.8 \\
\hline $4 B$ & 14 & 7 & 160 & 41.3 \\
\hline $4 B$ & 15 & 7 & 162 & 41.5 \\
\hline $4 \mathrm{~B}$ & 16 & 7 & 163 & 40.6 \\
\hline $4 \mathrm{~B}$ & 17 & 7 & 164 & 41.2 \\
\hline $4 \mathrm{~B}$ & 18 & 7 & 164 & 40.8 \\
\hline $4 \mathrm{~B}$ & 19 & 7 & 164 & 40.4 \\
\hline $4 \mathrm{~B}$ & 20 & 7 & 162 & 39.8 \\
\hline $4 \mathrm{~B}$ & 21 & 7 & 162 & 38.6 \\
\hline $4 \mathrm{~B}$ & 22 & 7 & 174 & 30.7 \\
\hline $4 B$ & 23 & 7 & 172 & 29.2 \\
\hline $4 \mathrm{~B}$ & 24 & 7 & 149 & 6.4 \\
\hline $4 B$ & 25 & 7 & 149 & 6.8 \\
\hline
\end{tabular}

the Forestadent Memory screw contains NiTi springs within the RPE body, which allows a more continuous, uniform expression of the force, reflected in the flatter

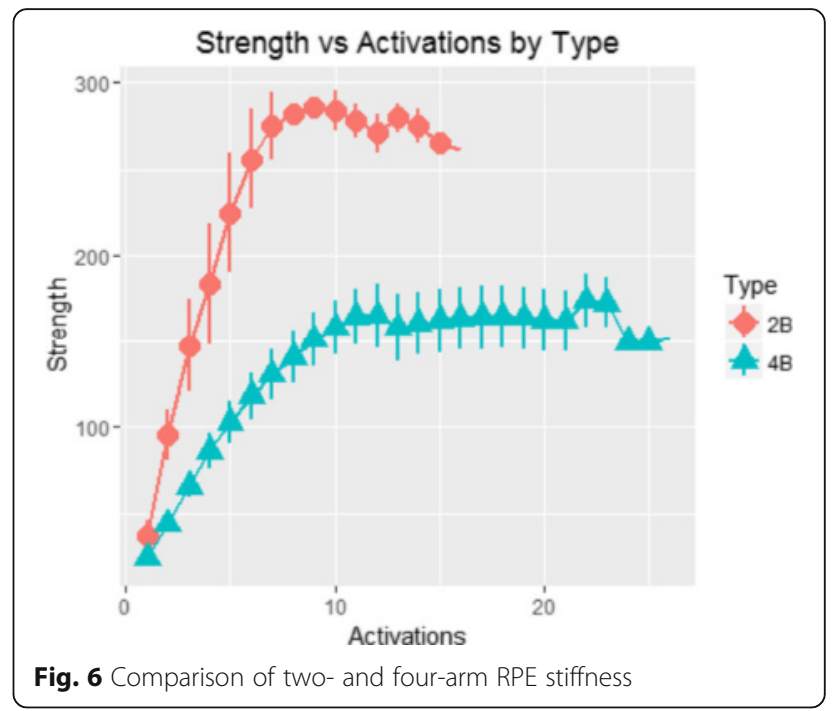

curve up to the maximum force of $212 \mathrm{~N}$, at which the screw blocks.

According to our results, the stiffest four-arm screw is the Leone A2620-10, which features telescopic guides, at a maximum force of $227 \mathrm{~N}$, followed by the Dentaurum Hyrax Click, which expressed a maximum force of 200.2 N. The other RPEs present curves characterized by a quasi-linear trend up to the ninth or tenth activation, followed by a steady reduction down to a plateau at which the force remains almost constant as the activations progress. Among these, that which developed the highest maximum force was the Lancer Philosophy 1 (179.9 N), followed by the Leone A0630-10 with orthogonal arms $(157.5 \mathrm{~N})$, the Forestadent Type $\mathrm{S}$ for narrow palates $(148.6 \mathrm{~N})$, and, finally, the Forestadent Anatomic Expander (124 N).

Table 3 and Fig. 6 show the means and standard deviations expressed by the two- and four-arm RPEs during activations.

The two-arm RPEs assessed in this study expressed more than double the force of their four-arm counterparts, even at five activations $(224 \pm 59.9 \mathrm{~N}$ vs. $103 \pm$

Table 4 Growth curve analysis coefficients

\begin{tabular}{lllll}
\hline Term & Estimate & Std. error & Statistic & Significance \\
\hline$a$ & -47 & 21 & -2.2 & $*$ \\
$\beta$ & 85 & 6.9 & 12 & $*$ \\
$\gamma$ & -7.1 & 0.96 & -7.4 & $*$ \\
$\delta$ & 0.19 & 0.04 & 4.8 & $*$ \\
$4 b_{i}$ & 40 & 25 & 1.6 & \\
$\beta_{4 b}$ & -56 & 7.3 & -7.7 & $*$ \\
$\gamma_{4 b}$ & 5.5 & 0.99 & 5.6 & $*$ \\
$\delta_{4 b}$ & -0.16 & 0.04 & -4.1 & $*$
\end{tabular}

The model

was strength ${ }_{i t}=a_{i}+4 b_{i}+\beta \times t+\beta_{4 b} \times t+\gamma \times t^{2}+\gamma_{4 b} \times t^{2}+\delta \times t^{3}+\delta_{4 b} \times t^{3}+\varepsilon_{i t}$ * $=p$ value $<0.05$ 


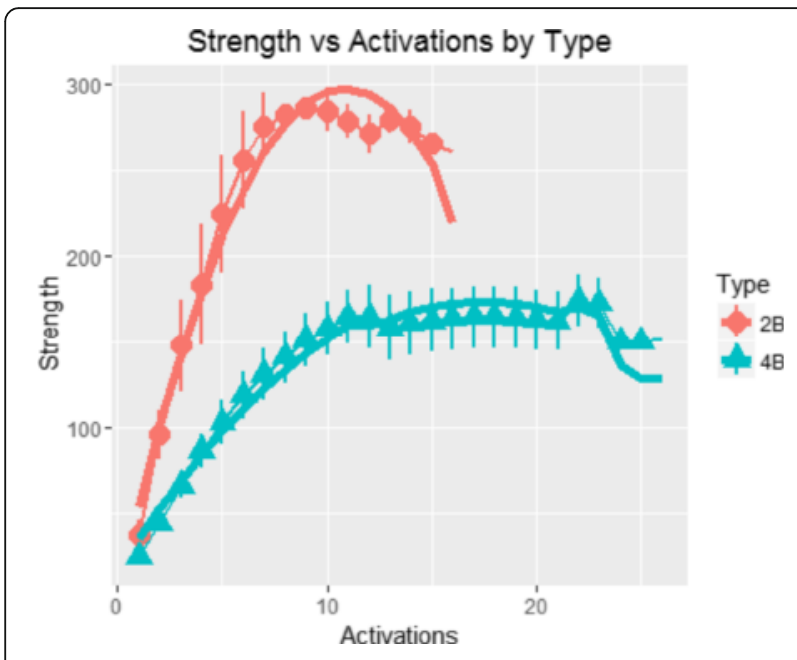

Fig. 7 Strength dynamics of the two- and four-arm RPE

32.9 N), maintaining far higher values as the activations progressed. Indeed, after 10 and 15 activations, the force remained over $250 \mathrm{~N}$, reflecting the conserved high level of stiffness. However, any more than 15 activations were prevented by structural deformations causing a block in the activation mechanism. In contrast, the four-arm RPEs continue to express a fairly constant force even after 20 activations, albeit at a much lower level.

The statistical analysis confirms the above descriptive considerations. The mixed-effect growth curve model coefficient estimates are shown in Table 4. In particular, the $t(t)$ statistically greater of two in absolute value indicates significant effects, and the coefficient table shows that:

- The linear term $\beta$ is positive, indicating an initial positive growth. The cubic term $\gamma$ is negative indicating that the increase in strength levels off as far as activation progresses. The cubic term $\delta$ is negative finally. All the three terms are statistically

Table 5 Model-specific intercept

\begin{tabular}{ll}
\hline & (Intercept) \\
\hline Dentaurum.Hyrax.Click.Medium & 37 \\
Dentaurum.Variety.SP & 1.1 \\
Forestadent.Anatomic.Expander.type.S & -39 \\
Forestadent.Anatomic.Expander.type.S.for.narrow.palates & -31 \\
Forestadent.Memory & 0.27 \\
Lancer.Philosophy.1 & 5.4 \\
Leone.A.0630.10.with.orthogonal.arms & -16 \\
Leone.A.2620.10.with.telescopic.guide & 44 \\
Leone.A.362113 & 13 \\
Target.Baby.REP.Veltri & -14 \\
\hline
\end{tabular}

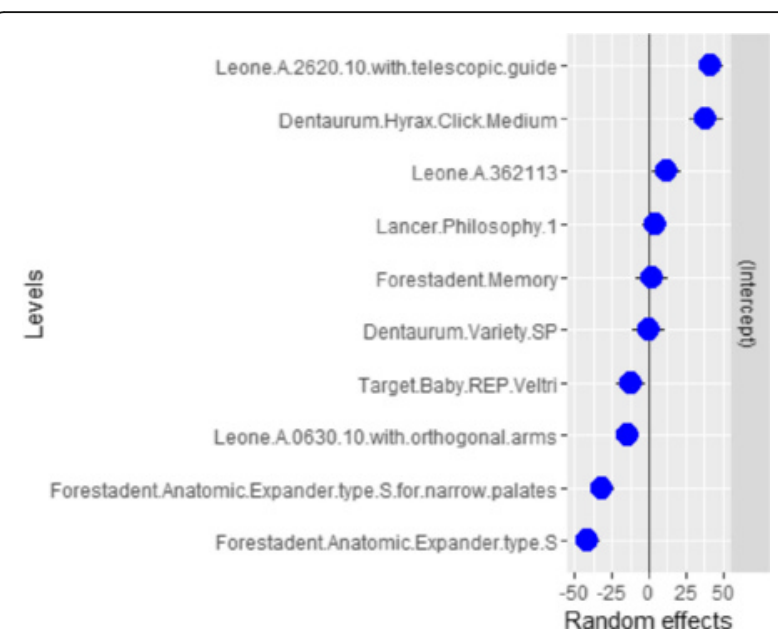

Fig. 8 Intercepts of the two- and four-arm RPE

significant indicating a non-linear behaviors of activation vs. strength.

- Activation and type $4 \mathrm{~b}$ interaction $\left(\beta_{\mathrm{ab}}\right)$ is negative and significant. This indicates that the initial increase is less steep for type $4 \mathrm{~b}$ models.

- All the higher polynomial terms interaction are significant $\left(\gamma_{\mathrm{ab}}, \delta_{\mathrm{ab}}\right)$, indicating that the curvature for the growth dynamic of type $4 \mathrm{~b}$ is different from that of the $2 \mathrm{~b}$ models.

Figure 7 shows that a three-degree polynomial can well approximate the strength dynamics in the observed range. Growth curve model-specific intercepts (random effect) are shown in Table 5 and Fig. 8. A higher intercept indicates a higher starting level.

Post hoc means comparison analysis was carried on the repeated measures ANOVA used to confirm growth curve analysis results. Figure 9 displays graphically the results showing that $2 \mathrm{~b}$ models consistently shows higher strength than $4 \mathrm{~b}$ ones from activations greater than 2.

During the course of the experiments, no breakage or deformation of any of the bands associated with any of the RPEs tested occurred.

\section{Discussion}

As shown in Figs. 10, 11, 12, and 13, the bending moments generated by the RPE arms were analyzed on both the horizontal and vertical planes. The greater stiffness of the two-arm RPEs may be linked to their smaller size, which allows them to be positioned on the same axis as the crowns of the molars used for anchorage. On the horizontal plane, this permits a point of application of the force to pass through the center of resistance of the system (which is determined by the expander as a whole, welded to the orthodontic bands), namely the anchoring 


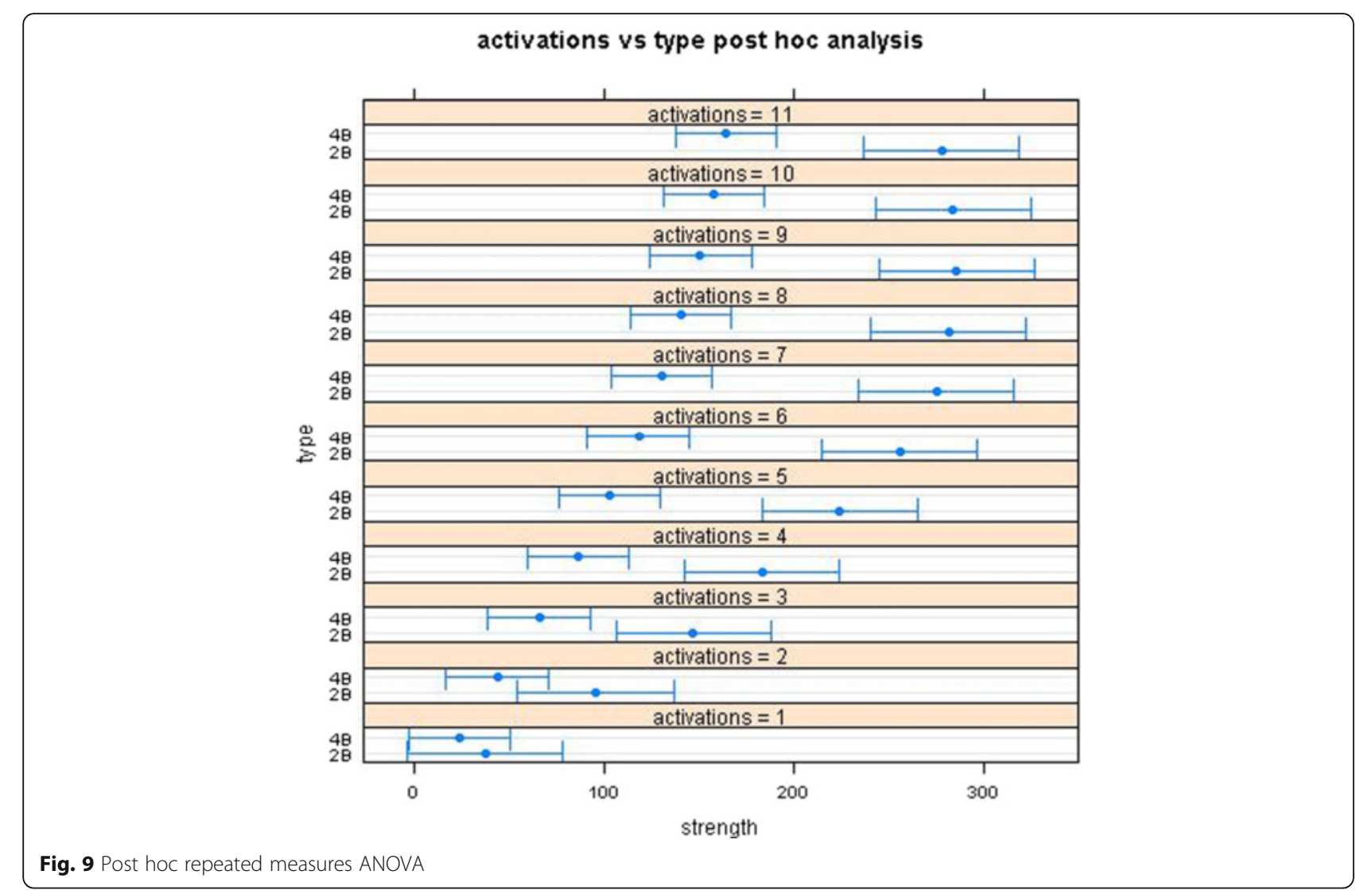

teeth on which the bands are cemented. Hence, the mechanical stresses have an optimal distribution on the structure of the screw, without the generation of bending moment (Fig. 10). This differs from the four-arm RPEs, whose screw position creates bending moment that tend to deform the screw body on the horizontal plane (Fig. 11). Such deformations increase with the number of activations, both due to the increase in force applied and the resulting lengthening of the screw, which reduces its stiffness.

On the vertical plane, the line of force expressed by the screw is at the same distance from the center of

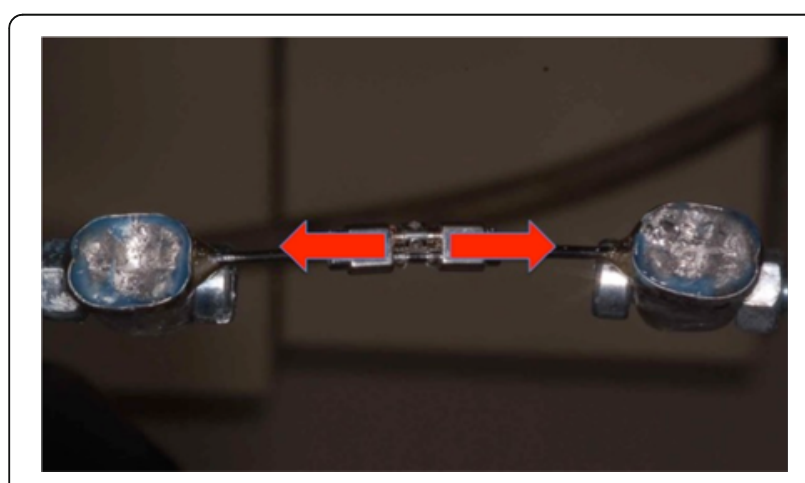

Fig. 10 Two-arm RPEs: no bending moment on the horizontal plane resistance in the two types of RPEs. However, the twoarm devices are susceptible to greater deformation as they have two fewer arms and lack the palatal support that opposes vertical deformation (Figs. 12 and 13). This deformation involves both the screw body and the retention arms and is responsible for the deterioration in the force expressed by the two-arm RPEs after the peak.

Within the four-arm category, the differences between those that express greater and lesser forces may be linked to the design of the arms, which are parallel to the guide in the former category and perpendicular in the latter. In this latter category, the arms are longer and have greater bends, which reduces their stiffness and therefore the force expressed (Fig. 14).

Few studies on this topic can be found in the literature. In a recent study, Muchitsch et al. [25] compared the stiffness of the retention arms of 16 commercially available RPE screws and found a difference in stiffness of $37.32 \%$. Camporesi et al., [26] on the other hand, measured the force expressed by three different fourarm RPEs, like us using the Instron machine, but without any bands or welding; they revealed maximum forces ranging between 215 and $156 \mathrm{~N}$. In contrast, Zimring and Isaacson [21], using intraoral dynamometers fixed to the RPEs mounted in the mouth of a sample of 5 patients, found forces ranging from 73 and 


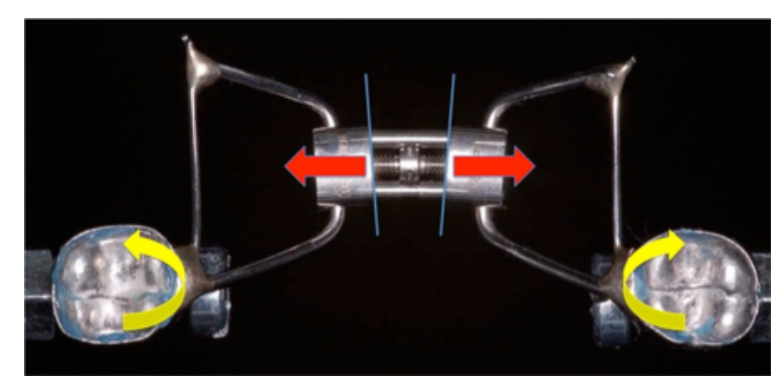

Fig. 11 Four-arm RPEs: bending moment generated on the horizontal plane

154 N. Sander et al. [34] also found smaller forces, between 70 and $120 \mathrm{~N}$, needed to activate a special precalibrated screw mounted in ten 9- to 13-year-old patients via a hyper-rapid activation protocol (one or two activations, five times a day).

As regards comparison of two- and four-arm types of RPEs, Lamparsky et al. [27] conducted a study to evaluate the difference in their clinical effects, using radiographs to quantify the separation of the median suture and plaster models to measure the inter-canine distance, inter-molar distance, and arch perimeter before expansion, after the active expansion phase and after removal of the RPE. Their results suggest that there is a little difference in the clinical effects on the median suture and teeth brought about by two- and four-arm RPEs.

In light of these studies, our in vitro results show that the forces expressed by RPEs welded to anchorage bands appear sufficient to separate the median palatine suture in pre-adolescent and adolescent patients, although the force expressed by certain models may be insufficient for this purpose in older patients. In particular, models Forestadent type $\mathrm{S}$ for narrow palates and Forestadent Anatomic Expander generate low maximum forces (respectively, 148.6 and $124 \mathrm{~N}$ ) that may be insufficient for the clinical demands reported elsewhere (Sander et al. [34]: 70-120 N, Zimring and Isaacson [21]: 73$154 \mathrm{~N})$. That being said, it is important to note that the

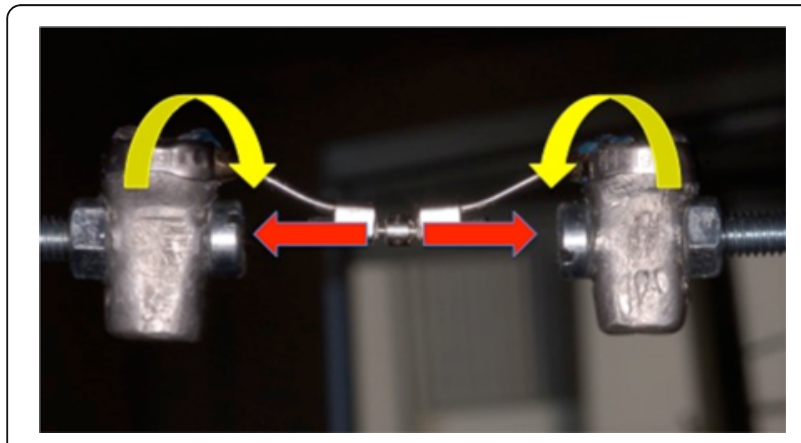

Fig. 12 Two-arm RPEs: bending moment generated on the vertical plane

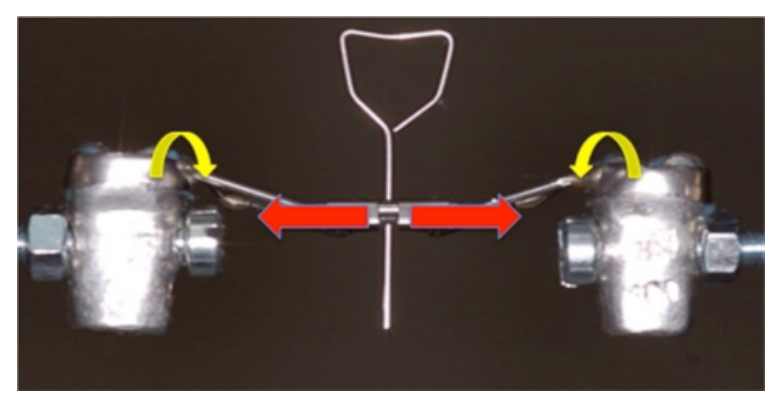

Fig. 13 Four-arm RPEs: bending moment generated on the vertical plane

stiffness in vivo will be strongly influenced by clinical factors such as the stiffness of the median palatine suture and the circummaxillary sutures, which is far lower than that of the Instron machine.

Recent literature is ever more frequently proposing the use of RPEs in adult patients, or using protocols of alternating expansion and contraction, which severely test the mechanical resistance of such devices [16-19]. Hence, manufacturers should take into account the mechanical stiffness of the RPEs being manufactured, as well as comfort, hygiene, and versatility issues. RPEs need to be manufactured in such a way as to maximize skeletal effects and minimize unwanted dentoalveolar effects. That being said, the two-arm models we tested showed a loss of force due to deformation way beyond the levels of force clinically required to separate the median palatine suture and therefore appear to be fit for purpose in terms of stiffness, in agreement with the findings of the clinical trial conducted by Bratu et al. [28].

\section{Conclusions}

The two-arm RPEs seem to be stiffer than their four-arm counterparts, and although from a mechanical perspective both are effective means of bringing about rapid expansion of the palate, certain models of the four-arm RPE may not express sufficient force to separate the median suture after puberty. The stiffness of the four-arm RPEs can be increased by using bands or bonding to fix them to the anterior teeth, but in addition to patient comfort, manufacturers should focus on the stiffness of both the retention arms
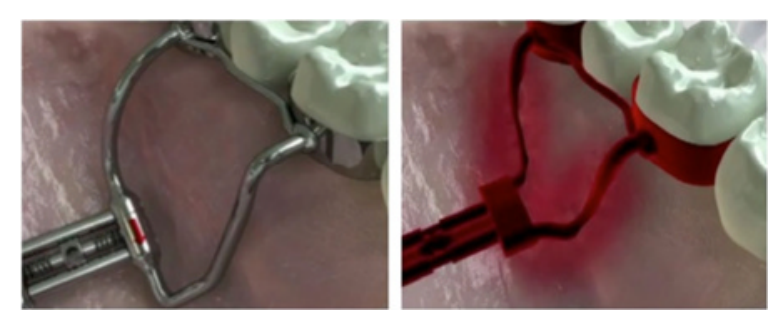

Fig. 14 Difference between RPEs with arms perpendicular and parallel to the guides 
and the screw body, whose propensity to generate a bending moment can be reduced by reducing its size. To this end, more research is needed into the resistance characteristics of RPEs, in particular to assess their suitability for older patients and alternating activation/contraction protocols and to determine how best to enhance skeletal and reduce unwanted dentoalveolar effects.

\section{Acknowledgements}

All authors made substantive contributions to the article and assumes full responsibility for its content.

\section{Authors' contribution}

All authors made substantive contributions to the article. LL and MF have conceived the design of the study. SE and MV have performed the tests and acquired the data. SGA has performed the statistical analysis. LM has drafted the manuscript and revised it critically. SG has given the final approval of the version to be published. All authors read and approved the final manuscript.

\section{Competing interests}

The authors declare that they have no competing interests.

\section{Consent for publication}

Written informed consent was obtained from the patient's parent for the publication of this report.

\section{Author details}

'Postgraduate School of Orthodontics, University of Ferrara, Via Montebello 31, 44121 Ferrara, Italy. ${ }^{2}$ Private Practice, Bologna, Italy. ${ }^{3}$ Department of Engineering, University of Ferrara, Via Saragat 1, 44122 Ferrara, Italy.

${ }^{4}$ UnipolSai Assicurazioni, Piazza della Costituzione 2, 40128 Bologna, Italy.

Received: 11 January 2016 Accepted: 3 October 2016

Published online: 28 November 2016

\section{References}

1. Di Malta E. Basi anatomo fisiologiche delle III classi: Terapia Ortopedica. Ed Martina. 2002;1:163-225.

2. D'Souza IM, Kumar HC, Shetty KS. Dental arch changes associated with rapid maxillary expansion: a retrospective model analysis study. Contemp Clin Dent. 2015:6(1):51-7.

3. Bishara SE, Stanley RN. Maxillary expansion: clinical implications. Am J Orthod Dentofacial Orthop. 1987:91:3-14.

4. Haas AJ. The treatment of maxillary deficiency by opening the midpalatal suture. Angle Orthod. 1965;35:200-17.

5. Haas AJ. Longterm post treatment evaluation of rapid palatal expansion. Angle Orthod. 1980;50:189-217.

6. Halicioğlu K, Yavuz I. Comparison of the effects of rapid maxillary expansion caused by treatment with either a memory screw or a Hyrax screw on the dentofacial structures_-transversal effects. Eur J Orthod. 2014:36(2):140-9.

7. Yurttadur G., Basciftci FA, Ozturk K. The effects of rapid maxillary expansion on voice function. Angle Orthod. 2016; [Epub ahead of print]

8. Ugolini A, Doldo T, Ghislanzoni LT, Mapelli A, Giorgetti R, Sforza C. Rapid palatal expansion effects on mandibular transverse dimensions in unilateral posterior crossbite patients: a three-dimensional digital imaging study. Prog Orthod. 2016:17:1.

9. Melsen B. Palatal growth studied on human autopsy material. A histologic microradiographic study. Am J Orthod. 1975;68:42-54.

10. Wertz R. Skeletal and dental changes accompanying rapid midpalatal suture opening. Am J Orthod. 1970;58:41-66.

11. Woller JL, Kim KB, Behrents RG, Buschng PH. An assessment of the maxilla after rapid maxillary expansion using cone beam computed tomography in growing children. Dental Press J Orthod. 2014;19(1):26-35.

12. Salgueiro DG, Rodrigues VH, Tieghi Neto V, Menezes CC, Gonçales ES, Ferreira JO. Evaluation of opening pattern and bone neoformation at median palatal suture area in patients submitted to surgically assisted rapid maxillary expansion (SARME) through cone beam computed tomography. J Appl Oral Sci. 2015;23(4):397-404.
13. Krebs A. Midpalatal suture expansion studied by the implant method over a seven-years period. Rep Congr Eur Orthod Soc. 1964:40:131-42.

14. Baccetti T, Franchi L, Cameron CG, McNamara Jr JA. Treatment timing for maxillary expansion. Angle Orthod. 2001;71(5):343-50.

15. Baccetti T, Franchi L, McNamara Jr JA. The cervical vertebral maturation (CVM) method for the assessment of optimal treatment timing in dentofacial orthopedics. Semin Orthod. 2005;11:119-29.

16. Liou EJ, Tsai WC. Maxillary protraction: a repetitive weekly protocol of alternate rapid maxillary expansions and constrictions. Cleft Palate Craniofac J. 2005:42:121-7.

17. Liou EJ. Effective maxillary orthopedic protraction for growing class III patients: a clinical application simulates distraction osteogenesis. Prog Orthod. 2005:6:154-71.

18. Franchi L, Baccetti T, Masucci C, Defraia E. Early Alt-RAMEC and facial mask protocol in class III malocclusion. J Clin Orthod. 2011:45:601-9.

19. Liu W, Zhou Y, Wang X, Liu D, Zhou S. Effect of maxillary protraction with alternating rapid palatal expansion and constriction vs expansion alone in maxillary retrusive patients: a single-center, randomized controlled trial. J Orthod Dentofacial Orthop. 2015;148(4):641-51.

20. Timms DJ. A study of basal movement with rapid maxillary expansion. Am J Orthod. 1980;77:500-7.

21. Zimring JF, Isaacson RJ. Forces produced by rapid maxillary expansion. III: forces present during retention. Angle Orthod. 1965;35:178-86.

22. Matsuyama Y, Motoyoshi M, Tsurumachi N, Shimizu N. Effects of palate depth, modified arm shape, and anchor screw on rapid maxillary expansion: a finite element analysis. Eur J Orthod. 2015;37(2):188-93.

23. Chen Y, Kim KA, Seo KW, Kang YG, Oh SH, Choi YS, Kim SH. A new designed expander supported by spike miniscrews with enhanced stability. J Craniofac Surg. 2016;27(2):e130-3.

24. Lin L, Ahn HW, Kim SJ, Moon SC, Kim SH, Nelson G. Tooth-borne vs boneborne rapid maxillary expanders in late adolescence. Angle Orthod. 2015; 85(2):253-62.

25. Muchitsch AP. Wendl B, Winsauer H, Pichelmayer M, Payer M. Rapid maxillary expansion screw on the test bench-a pilot study. Eur J Orthod. 2011;33:256-62.

26. Camporesi M, Franchi L, Doldo T, Defraia E. Evaluation of mechanical properties of three different screws for rapid maxillary expansion. BioMedical Engineering OnLine. 2013;12:128.

27. Lamparsky Jr DG, Rinchuse DJ, Close JM, Sciote JJ. Comparison of skeletal and dental changes between 2-point and 4-point rapid palatal expander. Am J Orthod Dentofacial Orthop. 2003;123:321-8.

28. Bratu DC, Bratu EA, Popa G, Luca M, Bälan R, Ogodescu A. Skeletal and dentoalveolar changes in the maxillary bone morphology using two-arm maxillary expander. Rom J Morphol Embryol. 2012:53(1):35-40.

29. Uysal T, Ramoglu SI, Ulker M, Ertas H. Effects of high-intensity curing lights on microleakage under orthodontic bands. Am J Orthod Dentofacial Orthop. 2010;138(2):201-7.

30. Mirman D. Growth curve analysis and visualization using R. CRC Press; 2016

31. R Core Team. R: a language and environment for statistical computing. Vienna: R Foundation for Statistical Computing; 2015. https://www.R-project. org/.

32. Bates $D$, Mächler M, Bolker B, Walker S. Fitting linear mixed-effects models using Ime4. J Stat Softw. 2015;67(1):1-48.

33. Lenth RV. Least-squares means: the R package Ismeans. J Stat Softw. 2016:69(1):1-33.

34. Sander C, Huffmeier S, Sander FM, Sander FG. Initial results regarding force exertion during rapid maxillary expansion in children. J Orofacial Orthop. 2006;67:19-26. 